\title{
JUSTIFICATION OF THE LINEAR LONG-WAVE APPROXIMATION TO VISCOUS FLUID FLOW DOWN AN INCLINED PLANE
}

\author{
BY \\ S. M. SUN AND M. C. SHEN \\ University of Wisconsin-Madison, Madison, Wisconsin
}

\begin{abstract}
The objective of this paper is to justify the linear long-wave approximation used in the derivation of approximate equations for long waves on the free surface of a two-dimensional viscous fluid flow down an inclined plane. To the first order of a small parameter, the approximate equation is a heat equation, which becomes ill-posed if a Reynolds number $R$ is greater than some critical value $R_{\mathrm{c}}$. To overcome this difficulty we consider a higher-order approximate equation, which is well-posed even if $R>R_{\mathrm{c}}$, and show that the solution of the higher-order equation is an approximation to the solution of the linearized Navier-Stokes equations. The justification is based upon a set of long-wave initial conditions, and the error bounds can also be expressed in terms of pointwise estimates.
\end{abstract}

1. Introduction. The problem of two-dimensional viscous fluid flow down an inclined plane has been investigated extensively in the past. The linear stability of a solution to this problem on the basis of a long-wave approximation was studied by Benjamin [1] and Yih [2] using the normal mode analysis, and a critical value $R_{\mathrm{c}}$ of the Reynolds number $R$ defined for this problem was found. The long waves on the viscous fluid down the plane are stable if $R<R_{\mathrm{c}}$ and unstable if $R>R_{\mathrm{c}}$. Later Mei [3], Benney [4], and others developed nonlinear theories for the evolution of long surface waves in this problem. In [3] $R$ was assumed small, and in [4] a two-parameter asymptotic expansion was developed to derive an evolution equation with high-order derivatives for long surface waves with an $R$ of order one and small amplitude. Up to now a mathematical justification of the asymptotic method used in the nonlinear theories remains an unsolved problem. If we choose a moving coordinate system at a speed $(R \sin \theta) / 2$ parallel to the plane where $\theta$ is the angle of inclination of the inclined plane and neglect nonlinear terms and all higher-order terms of a small parameter $\varepsilon$ in the evolution equation, a heat equation is obtained. The equation subject to an initial condition is ill-posed if $R>R_{\mathrm{c}}$ and well-posed if $R<R_{\mathrm{c}}$. Therefore, $R_{\mathrm{c}}$ is also associated with the so-called Hadamard instability of an ill-posed problem as recently coined by Joseph [5]. For $R<R_{\mathrm{c}}$, it has been rigorously justified by Shih and Shen [6] that a solution of the heat equation indeed 
is an approximation to the solution of the linear Navier-Stokes equations subject to the same initial condition of the free surface. For $R>R_{\mathrm{c}}$ Carasso and Shen [7] used a regularization method to study the ill-posed heat equation. However, a uniform error bound cannot be achieved in the justification of the method. Note that the linear Navier-Stokes equations are well-posed as will be shown later.

The objective of this paper is to justify rigorously an asymptotic method for the formal derivation of an evolution equation with a fourth-order derivative for the free surface, which is well-posed for $R>R_{\mathrm{c}}$ under less restrictive conditions. In this case we need not deal with an ill-posed problem and can establish uniform bounds for a solution of the equation with higher-order derivatives. Our main contribution is to show that a solution of this evolution equation is an approximation to the solution of the linear Navier-Stokes equations even if $R>R_{\mathrm{c}}$. The method of justification can be extended in a straightforward manner to approximate equations beyond the fourth-order equation. Here instead of using the formal long-wave stretching at the beginning, we impose a set of long-wave initial conditions and show that the longwave behavior persists in the solution of the linearized equations. Thus the validity of the long-wave approximation is clarified at least for the linear case.

We organize the paper as follows. In Sec. 2 the problem is formulated in a weak sense and several Hilbert spaces are defined for later use. In Sec. 3 we use a formal method [4] to derive the evolution equation with a fourth-order derivative. In Sec. 4 we prove our main theorem that under a set of assumptions the solution of the evolution equation indeed is an approximation to the solution of the linear NavierStokes equations and prove the error terms can be bounded by pointwise estimates.

2. Formulation. The fluid domain $\Omega$ that we are considering is a two-dimensional strip $\Omega$ : $-1<z<0,-\infty<x<+\infty$, bounded by the linearized free surface $\Gamma: z=0$, and a lower rigid plane $S: z=-1$. The $x$-axis is in the direction down the plane. Let $\theta$ be the angle of inclination, $0<\theta<\pi / 2$, and choose a coordinate system moving at the constant.speed $\lambda_{0}$ in the direction of the $x$-axis.

The equilibrium flow under gravity is one dimensional in the $x$-direction with a velocity given by

$$
u_{0}(z)=(R \sin \theta / 2)\left(1-z^{2}\right)-\lambda_{0},
$$

and the linearized Navier-Stokes equations governing the flow are

$$
\begin{gathered}
V_{t}+u_{0} V_{x}+V \cdot \nabla U=-\nabla p+R^{-1} \nabla^{2} V \quad \text { in } \Omega, \\
\nabla \cdot V=0, \\
\left(R^{-1} \mathscr{D}(V)-p\right) \cdot \bar{n}_{2}=\eta \sin \theta \bar{n}_{1}+\left(\sigma \eta_{x x}-\eta \cos \theta\right) \bar{n}_{2} \quad \text { on } \Gamma, \\
\eta_{t}+u_{0} \eta_{x}-w=0 \quad \text { on } \Gamma, \\
V=0 \quad \text { on } S, \\
V=V_{0}, \eta=\eta_{0} \quad \text { at } t=0 .
\end{gathered}
$$

Here $\bar{n}_{i}$ are the unit vectors of the coordinate system, a point is denoted by $(x, z)=$ $\left(x_{1}, x_{2}\right), t$ is the time, $V=\left(v_{1}, v_{2}\right)=(u, w)$ is the velocity, $U=\left(u_{0}, 0\right), p$ is the pressure, $\eta(x, t)$ is the deviation of the free surface from $z=0, R$ is the Reynolds 
number, $\sigma$ is the nondimensional surface tension coefficient, and

$$
\mathscr{D}(V)=\left[\frac{1}{2}\left(\frac{\partial v_{i}}{\partial x_{j}}+\frac{\partial v_{j}}{\partial x_{i}}\right)\right]
$$

denotes the deformation tensor. We note that all the variables have been nondimensionalized by appropriate units: $x, z$, and $\eta$ are measured in units of the equilibrium depth $h$ of the fluid, $t$ is measured in units of $(h / g)^{1 / 2}$ where $g$ is the constant gravitational acceleration, $u$ and $w$ are measured in units of $(g h)^{1 / 2}, p$ is measured in units of $\rho h g$ where $\rho$ is the density, $\sigma=s /\left(\rho g h^{2}\right)$ where $s$ is the surface tension coefficient, and

$$
R=\nu^{-1} h^{3 / 2} g^{1 / 2}
$$

where $\nu$ is the kinematic viscosity.

We will define several function spaces and state some definitions and lemmas. Let $J_{s}^{\infty}(\Omega)$ be the class of all solenoidal $C^{\infty}$-vector functions with compact support in $\Omega \cup \Gamma$ and $J_{s}(\Omega)$ be the Hilbert space obtained by completing $J_{s}^{\infty}(\Omega)$ with the scalar product

$$
(V, \Phi)=\int_{\Omega}\left(v_{1} \varphi_{1}+v_{2} \varphi_{2}\right) d A
$$

where $V=\left(v_{1}, v_{2}\right)$ and $\Phi=\left(\varphi_{1}, \varphi_{2}\right)$. The $L_{2}$-norm of a vector function $V$ on $\Omega$ is defined by

$$
\|V\|^{2}=\int_{\Omega}\left(v_{1}^{2}+v_{2}^{2}\right) d A
$$

It is known [8] that

$$
L_{2}(\Omega)=J_{s}(\Omega) \oplus G_{\Gamma}(\Omega)
$$

where $G_{\Gamma}(\Omega)$ are all vector functions of the form $\nabla p$ and $p$ is a single-valued locally square integrable scalar function on $\Omega$ and has first-order $L_{2}$-generalized derivatives with $p=0$ on $\Gamma$. Let $H(\Omega)$ be the Hilbert space obtained by completion of $J_{s}^{\infty}(\Omega)$ with respect to the norm

$$
\|V\|_{H}^{2}=\int_{\Omega} \sum_{i, j=1}^{2}\left(\frac{\partial v_{i}}{\partial x_{j}}\right)^{2} d A
$$

and $E(\Omega)$ be the completion of $J_{s}^{\infty}(\Omega)$ with respect to the energy norm

$$
\|V\|_{E}^{2}=\frac{1}{2} \int_{\Omega} \sum_{i, j=1}^{2}\left(\left(\frac{\partial v_{i}}{\partial x_{j}}\right)^{2}+\left(\frac{\partial v_{j}}{\partial x_{i}}\right)^{2}\right) d A
$$

and the scalar product

$$
E(V, \boldsymbol{\Phi})=\frac{1}{2} \int_{\Omega} \sum_{i, j=1}^{2}\left(\frac{\partial v_{i}}{\partial x_{j}}+\frac{\partial v_{j}}{\partial x_{i}}\right)\left(\frac{\partial \varphi_{i}}{\partial x_{j}}+\frac{\partial \varphi_{j}}{\partial x_{i}}\right) d A .
$$

Also we use $W_{2}^{m}(\Omega)$ and $W_{2}^{m}(\Gamma)$ to denote the standard Sobolev spaces for $m=$ $1,2,3, \ldots$ We denote by $L_{2}(T ; X)$ the class of functions $f:[0, T] \rightarrow X$ with 
the scalar product

$$
\int_{0}^{T}(f(t), g(t)) d t
$$

where $T$ is a fixed positive constant and $0 \leq t \leq T . C(T ; X)$ is the class of continuous functions with respect to the norm of $X$ and

$$
\|f(t)\|_{C(T ; X)}=\sup _{t \in[0, T]}\|f(t)\|_{X} .
$$

$C^{\prime}(T ; X)$ is the class of continuously strongly differentiable functions with

$$
\|f(t)\|_{C^{\prime}(T ; X)}=\sup _{t \in[0, T]}\|f(t)\|_{X}+\sup _{t \in[0, T]}\left\|f^{\prime}(t)\right\|_{X},
$$

where $f^{\prime}$ is the strong derivative of $f$. We say that $f \in L_{2}[T ; X]$ has a generalized derivative $f_{t} \in L_{2}[T ; X]$ if

$$
\int_{0}^{T}\left(f_{t}(t), g(t)\right) d t=-\int_{0}^{T}\left(f(t), g_{t}(t)\right) d t
$$

for all $g \in C^{\prime}(T ; X)$ with $g(0)=g(T)=0$. Next we state two lemmas, the proofs of which can be found in [6].

Lemma 1. $\|\cdot\|_{E}$ and $\|\cdot\|_{H}$ are equivalent and

$$
\left(1-2^{-1 / 2}\right)\|V\|_{H}^{2} \leq\|V\|_{E}^{2} \leq 2\|V\|_{H}^{2}
$$

if $V \in J_{s}^{\infty}(\Omega)$.

Lemma 2. For $V \in J_{s}^{\infty}(\Omega)$,

$$
\|V\| \leq 2^{3 / 2}\|V\|_{E} \quad \text { and } \quad\|V\|_{\Gamma} \leq 2^{3 / 2}\|V\|_{E}
$$

where

$$
\|V\|_{\Gamma}=\int_{\Gamma}\left(v_{1}^{2}+v_{2}^{2}\right) d x \text {. }
$$

We call $\left\{V(t, x, z)=\left(v_{1}, v_{2}\right)=(u, w), \eta(t, x)\right\}$ a generalized solution of Eqs. (1)-(6) if $V \in L_{2}(T ; E(\Omega)), V_{t} \in L_{2}(T ; E(\Omega)), \eta \in L_{2}\left(T ; L_{2}(\Gamma)\right), \eta_{t} \in$ $L_{2}\left(T ; L_{2}(\Gamma)\right), \eta_{x} \in L_{2}\left(T ; L_{2}(\Gamma)\right), w_{x} \in L_{2}\left(T ; L_{2}(\Gamma)\right)$, and $\{V, \eta\}$ satisfies Eqs. (4) and (6) and

$$
\begin{aligned}
\int_{0}^{T} d t & \int_{\Omega}\left(V_{t}+u_{0} V_{x}+V \cdot \nabla U\right) \cdot \Phi d A \\
= & \int_{0}^{T} d t \int_{\Gamma}\left(\eta \sin \theta \varphi_{1}-\sigma \eta_{x} \varphi_{2 x}-\eta \cos \theta \varphi_{2}\right) d x \\
& -(2 R)^{-1} \int_{0}^{T} d t \int_{\Omega} \mathscr{D}(V) \cdot \mathscr{D}(\Phi) d A,
\end{aligned}
$$

for all $\Phi=\left(\varphi_{1}, \varphi_{2}\right) \in L_{2}(T ; E(\Omega))$ and $\varphi_{2 x} \in L_{2}\left(T ; L_{2}(\Gamma)\right)$. We say the initial data $V_{0}, \eta_{0}$ in Eq. (6) are compatible with the free surface condition (3) if there exists a $p_{0} \in L_{2}(\Gamma)$ such that $V_{0}, \eta_{0}$, and $p_{0}$ satisfy (3). We also use

$$
D^{\beta}=\left(\frac{\partial^{\beta}}{\partial x^{\beta}}\right)
$$


where $\beta$ is a nonnegative integer. Sometimes we use $D_{x}$ to denote the $x$-derivative more explicitly.

Now we state a theorem, the proof of which can also be found in [6] or [9].

THEOREM 1. If the initial data $V_{0}$ and $\eta_{0}$ are compatible with the free surface condition (3) and if $V_{0}$ and $\eta_{0}$ possess generalized derivatives with respect to $x$ of all orders such that $D^{\beta} V_{0} \in E(\Omega) \cap W_{2}^{2}(\Omega)$ and $D^{\beta} \eta_{0} \in L_{2}(\Gamma)$ for all $\beta$ in $0 \leq \beta<\infty$, then there exists a unique generalized solution $\{V, \eta\}$ of Eqs. (1)-(6) such that

$$
\begin{array}{rlrl}
D^{\beta} V \in C(T ; E(\Omega)), & & D^{\beta} V_{t} \in L_{2}(T ; E(\Omega)), \\
D^{\beta} \eta \in C\left(T ; L_{2}(\Gamma)\right), & D^{\beta} \eta_{t} \in L_{2}\left(T ; L_{2}(\Gamma)\right),
\end{array}
$$

for all $T>0$ and any $\beta \geq 0$. Moreover, for each $\beta,\left\{D^{\beta} V, D^{\beta} \eta\right\}$ is the generalized solution of Eqs. (1)-(6) corresponding to the initial data $\left\{D^{\beta} V_{0}, D^{\beta} \eta_{0}\right\}$.

Note here that since the equations (1)-(6) are linear equations with coefficients independent of $x$, by letting $\Phi=V$ in (7) and straightforward estimates, we have

$$
\begin{aligned}
\|V(t)\|^{2} & +\cos \theta\|\eta(t)\|^{2}+\sigma\|\eta(t)\|_{H}^{2} \\
\leq & \|V(0)\|^{2}+\cos \theta\|\eta(0)\|^{2}+\sigma\|\eta(0)\|_{H}^{2} \\
& \quad+M \int_{0}^{t}\left(\|V(s)\|^{2}+\cos \theta\|\eta(s)\|^{2}+\sigma\|\eta(s)\|_{H}^{2}\right) d s,
\end{aligned}
$$

where $M$ is a positive constant. By the Gronwall inequality

$$
\|V(t)\|^{2}+\cos \theta\|\eta(t)\|^{2}+\sigma\|\eta(t)\|_{H}^{2} \leq e^{M t}\left(\|V(0)\|^{2}+\cos \theta\|\eta(0)\|^{2}+\sigma\|\eta(0)\|_{H}^{2}\right) .
$$

Therefore, Eqs. (1)-(6) always pose a well-posed problem in $0 \leq t \leq T$.

3. Formal asymptotic expansion. Let $\varepsilon$ be a small positive parameter, which measures the ratio of the length scale in the $z$-direction to that in the $x$-direction. Assume $\left\{V_{0}, \eta_{0}\right\}$ has the property that

$$
D^{k} V_{0}=O\left(\varepsilon^{k}\right), \quad D^{k} \eta_{0}=O\left(\varepsilon^{k}\right) \quad \text { for } k \geq 0,
$$

and let $\{V, \eta, \rho\}$ be the solution of Eqs. (1)-(6) corresponding to the initial data $\left\{V_{0}, \eta_{0}\right\}$. We suppose $\{V, p\}$ has the property $\partial / \partial x=O(\varepsilon)$ and $\partial / \partial t=O\left(\varepsilon^{3}\right)$ and $\{V, p\}$ possesses an asymptotic expansion of the form

$$
\varphi=\varphi_{1}+\varphi_{2}+\varphi_{3}+\cdots,
$$

where $\varphi_{1}=O(1), \varphi_{2}=O(\varepsilon), \ldots$ when $\varphi$ stands for $u$ and $p$ and $\varphi_{1}=O(\varepsilon)$, $\varphi_{2}=O\left(\varepsilon^{2}\right), \ldots$ when $\varphi$ stands for $w$. For the time being, $R$ and $\theta$ are fixed. Substituting Eq. (9) into Eqs. (1)-(6) and comparing orders of $\varepsilon$, we obtain the equations for the first-order approximations and solve for $u_{1}, w_{1}, p_{1}$, and $\lambda_{0}$ to have

$$
\begin{aligned}
u_{1}(t, x, z) & =\bar{u}_{1}(z) \eta(t, x)=(R \sin \theta)(1+z) \eta(t, x), \\
w_{1} & =\bar{w}_{1}(z) \eta_{x}=(R \sin \theta / 2)(1+z)^{2} \eta_{x}(t, x), \\
p_{1} & =\bar{p}_{1}(z) \eta=\eta(t, x) \cos \theta .
\end{aligned}
$$


Here we have not used Eq. (4) yet. Then from the second-, third-, and fourth-order approximation, we have

$$
\begin{aligned}
u_{2} & =\bar{u}_{2}(z) \eta_{x}, \quad p_{2}=\bar{p}_{2}(z) \eta_{x}, \quad w_{2}=\bar{w}_{2}(z) \eta_{x x}, \\
u_{3} & =\bar{u}_{3}(z) \eta_{x x}, \quad p_{3}=\bar{p}_{3}(z) \eta_{x x}, \quad w_{3}=\bar{w}_{3}(z) \eta_{x x x}, \\
u_{4} & =\bar{u}_{4}(z) \eta_{x x x}+\bar{u}_{41}(z) \eta_{t}, \quad p_{4}=\bar{p}_{4}(z) \eta_{x x x}+\bar{p}_{41}(z) \eta_{t}, \\
w_{4} & =\bar{w}_{4}(z) \eta_{x x x x}+\bar{w}_{41}(z) \eta_{x t} .
\end{aligned}
$$

The coefficients of $\eta$ and its derivatives in the successive approximations are complicated polynomials of $z$ distinguished by a bar with coefficients dependent only on $R, \theta, \sigma$ and will not be given. We substitute $w=w_{1}+w_{2}+w_{3}+w_{4}+\cdots$ into Eq. (4) and simplify it to obtain

$$
\begin{aligned}
\eta_{t}+\left(R \sin \theta-\lambda_{0}\right) \eta_{x}- & \left((R \sin \theta / 2) \eta_{x}+\bar{w}_{2}(0) \eta_{x x}\right. \\
+ & \left.\bar{w}_{3}(0) \eta_{x x x}+\bar{w}_{4}(0) \eta_{x x x x}+\bar{w}_{41}(0) \eta_{x t}\right)+w^{*}(t, x)=0,
\end{aligned}
$$

where $w^{*}(t, x)$ consists of higher-order terms of $w(t, x)$. We let $\lambda_{0}=(R \sin \theta) / 2$, omit $w^{*}$, remove $\eta_{x t}$ by the space derivatives of $\eta$, and discard the higher-order terms to obtain

$$
\eta_{1 t}+A_{1} \eta_{1 x x}+B_{1} \eta_{1 x x x}+C_{1} \eta_{1 x x x x}=0
$$

where $\eta_{1}$ is formally an approximation of $\eta$ and

$$
\begin{aligned}
& A_{1}=(R / 3)\left((2 / 5) R^{2} \sin ^{2} \theta-\cos \theta\right), \\
& B_{1}=R \sin \theta+(10 / 21) R^{2} A_{1} \sin \theta, \\
& C_{1}=(\sigma / 3)+R\left[(75872 / 2027025) R^{6} \sin ^{4} \theta-(17363 / 155925) R^{4} \sin ^{2} \theta \cos \theta\right. \\
& \left.\quad+(157 / 224) R^{2} \sin ^{2} \theta-(8 / 15) \cos \theta+(2 / 45) R^{2} \cos \theta\right] .
\end{aligned}
$$

The detailed derivation related to Eq. (10) in the much more general case that includes all nonlinear terms and also a magnetic field in the fluid region was given in [10]. One can also check easily that (10) is equivalent to Eq. (46) in [4] except that in our formulation a different definition of $R$ was used. Shih and Shen [6] assumed $A_{1}<0$ and proved that the solution of

$$
\eta_{1 t}+A_{1} \eta_{1 x x}=0
$$

is a long-wave approximation to a solution of Eqs. (1)-(6). But if $A_{1}>0,(11)$ becomes an ill-posed problem. Therefore, (11) is no longer valid for $A_{1} \geq 0$ and a regularization method was developed to study the ill-posed problem [7]. Here instead we include higher-order terms to get a meaningful equation (10). In the following we assume $A_{1} \geq 0$ and $C_{1}>0$. Note that $C_{1}>0$ if $\sigma$ or $R$ is sufficiently large. Thus if $C_{1}>0$, then Eq. (10) coupled with the initial condition

$$
\eta_{1}(0, x)=\eta_{0}(x) \quad \text { at } t=0
$$

is a well-posed problem and possesses a unique solution $\eta_{1}$. Therefore, we can obtain formal approximations of $u, w$, and $p$. Next we prove that these approximations are indeed approximate solutions of Eqs. (1)-(6) for the long-wave initial data. 
4. Asymptotic approximate solution. We shall show that $\eta_{1}$ in Eq. (10) with the initial condition

$$
\eta_{1}(0, x)=\eta_{0}(x) \quad \text { at } t=0
$$

is an approximation of $\eta$ and make the following assumptions:

A.1. $0<\theta<(\pi / 2), C_{1}>0$, and $A_{1} \geq 0$.

A.2. The initial data $V_{0}$ and $\eta_{0}$ possess generalized derivatives with respect to $x$ of all orders such that $D^{\beta} V_{0} \in E(\Omega) \cap W_{2}^{2}(\Omega), D^{\beta} \eta_{0} \in L_{2}(\Gamma)$ for all $\beta \geq 0$ and $V_{0}$, $\eta_{0}$ are compatible with the free surface conditions (3).

A.3. Long-wave condition. There exists a positive parameter $\varepsilon$ such that for $0<\varepsilon<1$, the initial data $V(0), \eta(0), V_{b}(0)$ for $V, \eta, V_{b}$ satisfy

$$
\left\|D^{\beta} \eta(0)\right\| \leq \varepsilon^{\beta}, \quad\left\|D^{\beta}\left(V(0)-V_{b}(0)\right)\right\| \leq \varepsilon^{2+\beta},
$$

for all $\beta \geq 0$, where $V_{b}=\left(u_{b}, w_{b}\right)=\left(\bar{u}_{1}(z) \eta_{1}+\bar{u}_{2}(z) \eta_{1 x}, \bar{w}_{1}(z) \eta_{1 x}+\bar{w}_{2}(z) \eta_{1 x x}\right)$.

Then we shall prove

Theorem 2. There exists a positive number $\varepsilon_{0}$ which depends only on $A_{1}, B_{1}, C_{1}$, $R, \theta$, and $T$. If the initial data $V_{0}$ and $\eta_{0}$ satisfy the assumptions A.1 to A.3 and $0<\varepsilon<\varepsilon_{0}$, then the approximate solution $\left\{V_{b}, \eta_{1}\right\}$ which is defined in Eqs. (10) and (12) and A.3 is an asymptotic approximation to the generalized solution $\{V, \eta\}$ in the following sense:

$$
\left\|u-u_{b}\right\| \leq \varepsilon^{2} L_{1} e^{\delta t}, \quad\left\|w-w_{b}\right\| \leq \varepsilon^{3} L_{1} e^{\delta t}, \quad\left\|\eta-\eta_{1}\right\| \leq \varepsilon^{2} L_{1} e^{\delta t}
$$

for all $0 \leq t \leq T$, where $L_{1}$ and $\delta$ are positive constants depending on $\sigma, R, \theta$ and $\|\cdot\|$ is the usual $L_{2}$-norm on $\Omega$ or $\Gamma$.

We note here that $D^{\beta} V_{b}(0)$ is well defined and conditions in A.3 are not mutually independent. Since $V_{0}$ and $\eta_{0}$ satisfy the assumptions in Theorem 1, Eqs. (1)-(6) have a unique generalized solution $\{V, \eta\}$ corresponding to the initial data $\left\{V_{0}, \eta_{0}\right\}$ and $\left\{D^{\beta} V, D^{\beta} \eta\right\}$ is the generalized solution of Eqs. (1)-(6) corresponding to the initial data $\left\{D^{\beta} V_{0}, D^{\beta} \eta_{0}\right\}$. Let

$$
\begin{aligned}
V & =(u, w)=V_{a}+V_{i}+V^{*}, \\
u & =u_{a}+\bar{u}_{3}(z) \eta_{x x}+\bar{u}_{4}(z) \eta_{x x x}-\left(A_{1} \eta_{x x}+B_{1} \eta_{x x x}\right) \bar{u}_{41}(z)+u^{*}, \\
w & =w_{a}+\bar{w}_{3}(z) \eta_{x x x}+\bar{w}_{4}(z) \eta_{x x x x}-\left(A_{1} \eta_{x x x}+B_{1} \eta_{x x x x}\right) \bar{w}_{41}(z)+w^{*},
\end{aligned}
$$

where

$$
\begin{aligned}
V^{*} & =\left(u^{*}, w^{*}\right), \\
V_{a} & =\left(u_{a}, w_{a}\right)=\left(\bar{u}_{1}(z) \eta+\bar{u}_{2}(z) \eta_{x}, \bar{w}_{1}(z) \eta_{x}+\bar{w}_{2}(z) \eta_{x x}\right),
\end{aligned}
$$

and $V_{i}$ is the remainder. Since $V$ and $\eta$ are the generalized solutions of Eqs. (1)-(6), we substitute Eqs. (15) into Eq. (4) to get

$$
\begin{gathered}
\eta_{t}+A_{1} \eta_{x x}+B_{1} \eta_{x x x}+C_{1} \eta_{x x x x}=w^{*} \\
\eta=\eta_{0} \quad \text { at } t=0 .
\end{gathered}
$$


By substituting Eqs. (15) into Eq. (7) and choosing $\Phi$ in such a form that $\Phi(s)=0$ for $t \leq s \leq T$, we have

$$
\begin{gathered}
\int_{0}^{t} d s \int_{\Omega}\left[V_{a t}+V_{i t}+V_{t}^{*}+u_{0}\left(V_{a x}+V_{i x}+V_{x}^{*}\right)+\left(V_{a}+V_{i}+V^{*}\right) \cdot \nabla U\right] \cdot \Phi d A \\
=\int_{0}^{t} d s \int_{\Gamma}\left(\eta \varphi_{1} \sin \theta-\sigma \eta_{x} \varphi_{2 x}-\eta \varphi_{2} \cos \theta\right) d x \\
-(2 R)^{-1} \int_{0}^{t} d s \int_{\Omega}\left[\mathscr{D}\left(V_{a}+V_{i}+V^{*}\right)\right] \cdot \mathscr{D}(\Phi) d A .
\end{gathered}
$$

Since $\eta$ possesses generalized derivatives with respect to $x$ of all orders, we can use integration by parts to simplify Eq. (19) to obtain

$$
\begin{aligned}
\int_{0}^{t} d s & \int_{\Omega}\left(V_{t}^{*}+u_{0} V_{x}^{*}+V^{*} \cdot \nabla U\right) \cdot \Phi d A \\
= & \int_{0}^{t} d s \int_{\Gamma}\left(G \cdot \phi-\sigma \eta_{x} \varphi_{2 x}\right) d x \\
& +\int_{0}^{t} d s \int_{\Omega} F \cdot \Phi d A-(2 R)^{-1} \int_{0}^{t} d s \int_{\Omega} \mathscr{D}\left(V^{*}\right) \cdot \mathscr{D}(\Phi) d A,
\end{aligned}
$$

where $F$ and $G$ can be expressed as follows:

$$
\begin{aligned}
G= & \left(G_{0}+G_{1} D_{x}+G_{2} D_{x}^{2}\right) \eta_{x x x}, \\
F= & \left(F_{0}+F_{1} D_{x}+F_{2} D_{x}^{2}+F_{3} D_{x}^{3}\right) \eta_{x x x} \\
& +\left(E_{0}+E_{1} D_{x}+E_{2} D_{x}^{2}+E_{3} D_{x}^{3}+E_{4} D_{x}^{4}\right) \eta_{t} .
\end{aligned}
$$

Note that $F_{0}, \ldots, F_{3}, G_{0}, \ldots, G_{2}$, and $E_{0}, \ldots, E_{4}$ are vector functions of $z$ only related to $\bar{u}_{i}, \bar{w}_{i}$, and $\bar{p}_{i}$.

Before proving Theorem 2, we begin by proving a series of lemmas. In the following, $C_{i}$ for $i \neq 1$ and $K_{i}$ will denote positive constants depending upon $R, \theta$, and $\sigma$ only.

Lemma 3. There exist positive constants $C_{0}, C_{2}$ such that

$$
\begin{aligned}
\int_{0}^{t}\left\|D^{\beta} V^{*}\right\|_{E}^{2} d s \leq & C_{0}\left\|D^{\beta} V^{*}(0)\right\|^{2} \\
& +C_{2} \exp (\nu t)\left(\sum_{i=2}^{5}\left\|D^{\beta} D_{x}^{i} \eta(0)\right\|^{2}+\int_{0}^{t}\left(\sum_{i=1}^{4}\left\|D^{\beta} D_{x}^{i} V^{*}\right\|_{E}^{2}\right) d s\right)
\end{aligned}
$$

for all $\beta \geq 0$ and any $t \geq 0$ and $\nu$ is a fixed positive constant.

Proof. Replacing $\Phi$ in Eq. (20) by $V^{*}$ and using $\int u_{0} V_{x}^{*} V d \Omega=0$, we have

$$
\begin{aligned}
\frac{1}{2}\left(\left\|V^{*}(t)\right\|^{2}-\left\|V^{*}(0)\right\|^{2}\right)= & -\int_{0}^{t} d s \int_{\Omega} u_{0 z} w^{*} u^{*} d A+\int_{0}^{t} d s \int_{\Omega} F \cdot V^{*} d A \\
& +\int_{0}^{t} d s \int_{\Gamma} G \cdot V^{*} d x-R^{-1} \int_{0}^{t}\left\|V^{*}\right\|_{E} d s .
\end{aligned}
$$


By Lemmas 1 and 2, integration by parts, and noting that $\int_{\Omega}\left|w^{*}\right|^{2} d A \leq K\left\|V_{x}^{*}\right\|_{E}^{2}$ since $w^{*}=\int_{0}^{z} w_{z}^{*} d z=-\int_{0}^{z} u_{x}^{*} d z$, we have the estimates

$$
\begin{aligned}
\left|\int_{\Omega} u_{0 z} w^{*} u^{*} d A\right| & \leq K_{1}\left\|V^{*}\right\|_{E}\left\|V_{x}^{*}\right\|_{E} \\
\left|\int_{\Omega} F \cdot V^{*} d A\right| & \leq K_{2}\left(\sum_{i=3}^{5}\left\|D_{x}^{i} \eta\right\|_{E}^{2}+\sum_{i=0}^{3}\left\|D_{t} D_{x}^{i} \eta\right\|^{2}\right)^{1 / 2}\left\|V^{*}\right\|_{E} \\
\left|\int_{\Gamma} G \cdot V^{*} d A\right| & \leq K_{3}\left(\sum_{i=3}^{5}\left\|D_{x}^{i} \eta\right\|^{2}\right)^{1 / 2}\left\|V^{*}\right\|_{E} .
\end{aligned}
$$

Therefore,

$$
\begin{aligned}
&\left\|V^{*}(t)\right\|^{2}-\left\|V^{*}(0)\right\|^{2} \\
& \leq 2 K_{1} \int_{0}^{t}\left\|V^{*}\right\|_{E}\left\|V_{x}^{*}\right\|_{E} d s \\
&+K_{4} \int_{0}^{t}\left(\sum_{i=3}^{5}\left\|D_{x}^{i} \eta\right\|^{2}+\sum_{i=0}^{3}\left\|D_{t} D_{x}^{i} \eta\right\|^{2}\right)^{1 / 2}\|V\|_{E} d s-2 R^{-1} \int_{0}^{t}\left\|V^{*}\right\|_{E}^{2} d s \\
& \leq K_{5} \int_{0}^{t}\left\|V_{x}^{*}\right\|_{E} d s+K_{6} \int_{0}^{t}\left(\sum_{i=3}^{5}\left\|D_{x}^{i} \eta\right\|^{2}+\sum_{i=0}^{3}\left\|D_{t} D_{x}^{i} \eta\right\|^{2}\right)^{1 / 2} d s \\
&-R^{-1} \int_{0}^{t}\left\|V^{*}\right\|_{E}^{2} d s
\end{aligned}
$$

where $D_{t}=\partial / \partial t$. Thus

$$
\begin{aligned}
\int_{0}^{t}\left\|V^{*}\right\|_{E}^{2} d s \leq & R\left\|V^{*}(0)\right\|^{2}+R K_{5} \int_{0}^{t}\left\|V_{x}^{*}\right\|_{E}^{2} d s \\
& +R K_{6} \int_{0}^{t}\left(\sum_{i=3}^{5}\left\|D_{x}^{i} \eta\right\|^{2}+\sum_{i=0}^{3}\left\|D_{t} D_{x}^{i} \eta\right\|^{2}\right)^{1 / 2} d s .
\end{aligned}
$$

But from Eq. (17), we have

$$
\begin{aligned}
\int_{\Gamma}\left(\eta_{t}+\right. & \left.A_{1} \eta_{x x}+B_{1} \eta_{x x x}+C_{1} \eta_{x x x x}\right)^{2} d x \\
= & \int_{\Gamma}\left|w^{*}\right|^{2} d x \\
= & \left\|\eta_{t}\right\|^{2}+A_{1}^{2}\left\|\eta_{x x}\right\|^{2}+B_{1}^{2}\left\|\eta_{x x x}\right\|^{2}+C_{1}^{2}\left\|\eta_{x x x x}\right\|^{2} \\
& \quad-\int_{\Gamma}\left(A_{1}\left(\eta_{x}^{2}\right)_{t}-C_{1}\left(\eta_{x x}^{2}\right)_{t}-2 B_{1} \eta_{t} \eta_{x x x}\right) d x-2 A_{1} C_{1}\left\|\eta_{x x x}\right\|^{2}
\end{aligned}
$$


and

$$
\begin{gathered}
\int_{0}^{t}\left(\left\|\eta_{t}\right\|^{2}+A_{1}^{2}\left\|\eta_{x x}\right\|^{2}+\left(B_{1}^{2}-2 A_{1} C_{1}\right)\left\|\eta_{x x x}\right\|^{2}+C_{1}^{2}\left\|\eta_{x x x x}\right\|^{2}\right) d s \\
-A_{1}\left\|\eta_{x}(t)\right\|^{2}+A_{1}\left\|\eta_{x}(0)\right\|^{2}+C_{1}\left\|\eta_{x x}(t)\right\|^{2}-C_{1}\left\|\eta_{x x}(0)\right\| \\
+2 B_{1} \int_{0}^{t} d s \int_{\Gamma} \eta_{t} \eta_{x x x} d x=\int_{0}^{t} d s \int_{\Gamma}\left|w^{*}\right|^{2} d x
\end{gathered}
$$

Multiply Eq. (17) by $\eta_{x x x}$ to obtain

$$
\int_{0}^{t} d s \int_{\Gamma}\left(\eta_{t} \eta_{x x x}+B_{1} \eta_{x x x}^{2}\right) d x=\int_{0}^{t} d s \int_{\Gamma} \eta_{x x x} w^{*} d x
$$

Then multiply Eq. (17) by $\eta_{x x}$ to obtain

$$
\begin{aligned}
& \left\|\eta_{x}(t)\right\|^{2}-\left\|\eta_{x}(0)\right\|^{2} \\
& \quad=A_{1} \int_{0}^{t}\left\|\eta_{x x}\right\|^{2} d s-C_{1} \int_{0}^{t}\left\|\eta_{x x x}\right\|^{2} d s-\int_{0}^{t} d s \int_{\Gamma} w^{*} \eta_{x x} d x .
\end{aligned}
$$

From Eqs. (25)-(27) we have

$$
\begin{gathered}
\int_{0}^{t}\left(\left\|\eta_{t}\right\|^{2}-\left(B^{2}+A_{1} C_{1}\right)\left\|\eta_{x x x}\right\|^{2}+C_{1}^{2}\left\|\eta_{x x x x}\right\|^{2}\right) d s \\
+C_{1}\left\|\eta_{x x}(t)\right\|^{2}-C_{1}\left\|\eta_{x x}(0)\right\|+2 B_{1} \int_{0}^{t} d s \int_{\Gamma} \eta_{x x x} w^{*} d x \\
=\int_{0}^{t} d s \int_{\Gamma}\left|w^{*}\right|^{2} d x-A_{1} \int_{0}^{t} d s \int_{\Gamma} w^{*} \eta_{x x} d x
\end{gathered}
$$

By using $w^{*}=\int_{0}^{z} w_{z}^{*} d z=-\int_{0}^{z} u_{x}^{*} d z$,

$$
\begin{gathered}
\int_{0}^{t}\left(\left\|\eta_{t}\right\|^{2}+C_{1}^{2}\left\|\eta_{x x x x}\right\|^{2}\right) d s+C_{1}\left\|\eta_{x x}(t)\right\|^{2}-C_{1}\left\|\eta_{x x}(0)\right\|^{2} \\
\leq K_{7} \int_{0}^{t}\left(\left\|\eta_{x x}\right\|^{2}+\left\|\eta_{x x x}\right\|^{2}\right) d s+K_{8} \int_{0}^{t}\left\|V_{x}^{*}\right\|_{E}^{2} d s
\end{gathered}
$$

But

$$
\int_{\Gamma} \eta_{x x x}^{2} d x \leq \frac{1}{2}\left(\rho \int_{\Gamma} \eta_{x x x x}^{2} d x+\frac{1}{\rho} \int_{\Gamma} \eta_{x x}^{2} d x\right)
$$

by the usual interpolation inequalities where $\rho$ is any small constant. Hence,

$$
\begin{gathered}
\int_{0}^{t}\left(\left\|\eta_{t}\right\|^{2}+\left(C_{1}^{2}-\frac{\rho}{2}\right)\left\|\eta_{x x x x}\right\|^{2}\right) d s+C_{1}\left\|\eta_{x x}(t)\right\|^{2}-C_{1}\left\|\eta_{x x}(0)\right\|^{2} \\
\leq K_{7}\left(1+\frac{1}{2 \rho}\right) \int_{0}^{t}\left\|\eta_{x x}\right\|^{2} d s+K_{8} \int_{0}^{t}\left\|V_{x}^{*}\right\|_{E}^{2} d s
\end{gathered}
$$

Let $\rho=C_{1}^{2}$, and by the Gronwall inequality

$$
\begin{aligned}
\int_{0}^{t}\left\|\eta_{x x}\right\|^{2} d s \leq & C_{1}^{-1} \exp \left(K_{7}\left(1+\frac{1}{2 \rho}\right) t C_{1}^{-1}\right)\left(C_{1}^{2}\left(K_{7}\left(1+(2 \rho)^{-1}\right)\right)^{-1}\left\|\eta_{x x}(0)\right\|^{2}\right. \\
& \left.+\int_{0}^{t} \exp \left(-K_{7}\left(1+\frac{1}{2 \rho}\right) \tau C_{1}^{-1}\right) K_{8}\left(\int_{0}^{\tau}\left\|V_{x}^{*}\right\|_{E}^{2} d s\right) d \tau\right) \\
\leq & K_{9} \exp \left(K_{10} t\right)\left(\int_{0}^{t}\left\|V_{x}^{*}\right\|_{E}^{2} d s+\left\|\eta_{x x}(0)\right\|^{2}\right)
\end{aligned}
$$


Therefore, by Eqs. (28) and (29)

$$
\begin{aligned}
& \int_{0}^{t}\left(\left\|\eta_{t}\right\|^{2}+\left\|\eta_{x x}\right\|^{2}+\left\|\eta_{x x x}\right\|^{2}+\left\|\eta_{x x x x}\right\|^{2}\right) d s \\
& \quad \leq K_{11}\left(\exp \left(K_{10} t\right)+1\right)\left(\int_{0}^{t}\left\|V_{x}^{*}\right\|_{E}^{2} d s+\left\|\eta_{x x}(0)\right\|^{2}\right) .
\end{aligned}
$$

We note here that $K_{i}, i=1,2, \ldots, 11$, are independent of $t$. Since Eqs. (17) and (22) hold for $D^{\beta} \eta, D^{\beta} V^{*}$ with $\beta \geq 0$, by the same derivation,

$$
\begin{gathered}
\int_{0}^{t}\left(\left\|D^{\beta} \eta_{t}\right\|^{2}+\left\|D^{\beta} \eta_{x x}\right\|^{2}+\left\|D^{\beta} \eta_{x x x}\right\|^{2}+\left\|D^{\beta} \eta_{x x x x}\right\|^{2}\right) d s \\
\leq K_{11}\left(\exp \left(K_{10} t\right)+1\right)\left(\left\|D^{\beta} \eta_{x x}(0)\right\|^{2}+\int_{0}^{t}\left\|D^{\beta} V_{x}^{*}\right\|_{E}^{2} d s\right), \\
\int_{0}^{t}\left\|D^{\beta} V^{*}\right\|_{E} d s \leq R\left\|D^{\beta} V^{*}(0)\right\|^{2}+R K_{5} \int_{0}^{t}\left\|D^{\beta} V_{x}^{*}\right\|_{E}^{2} d s \\
+R K_{6} \int_{0}^{t}\left(\sum_{i=3}^{5}\left\|D^{\beta} D_{x}^{i} \eta\right\|^{2}+\sum_{i=0}^{3}\left\|D_{t} D_{x}^{i} \eta\right\|^{2}\right)^{1 / 2} d s .
\end{gathered}
$$

Thus combining Eqs. (31) and (32), we have

$$
\begin{aligned}
\int_{0}^{t}\left\|D^{\beta} V^{*}\right\|_{E}^{2} d s \leq & C_{0}\left\|D^{\beta} V^{*}(0)\right\|^{2}+C_{3} \int_{0}^{t}\left\|D^{\beta} V_{x}^{*}\right\|_{E}^{2} d s \\
& +C_{4} \exp (\nu t)\left(\sum_{i=0}^{3}\left(\left\|D^{\beta} D_{x}^{i+2} \eta(0)\right\|^{2}+\int_{0}^{t}\left\|D^{\beta} D_{x}^{i+1} V^{*}\right\|_{E}^{2} d s\right)\right) \\
\leq & C_{0}\left\|D^{\beta} V^{*}(0)\right\|^{2} \\
& +C_{2} \exp (\nu t)\left(\sum_{i=1}^{4} \int_{0}^{t}\left\|D^{\beta} D_{x}^{i} V^{*}\right\|_{E}^{2} d s+\sum_{i=2}^{5}\left\|D^{\beta} D_{x}^{i} \eta(0)\right\|^{2}\right),
\end{aligned}
$$

for all $\beta \geq 0$. This proves the lemma.

Lemma 4. There exist two positive numbers $C_{2}$ and $\nu$ defined in Lemma 3 such that if $0<\varepsilon<\varepsilon_{0}=\left(\left(2 C_{2}\right)^{-1} \exp (-\nu t)\right)^{1 / 2}$, we have

$$
\int_{0}^{t}\left\|D^{\beta} V^{*}\right\|_{E}^{2} d s \leq C_{5} \varepsilon^{4+2 \beta} \exp (\nu t)(1-r)^{-1},
$$

where $r=\left(\varepsilon / \varepsilon_{0}\right)^{2}$ and $C_{5}$ depends on $R, \theta, \sigma$ only.

Proof. By A.3 and (15),

$$
\begin{aligned}
\left\|D^{\beta} V^{*}(0)\right\| & \leq\left\|D^{\beta}\left(V(0)-V_{a}(0)\right)\right\|+\left\|D^{\beta} V_{i}(0)\right\| \\
& \leq \varepsilon^{2+\beta}+K_{1} \varepsilon^{2+\beta} \leq\left(K_{1}+1\right) \varepsilon^{2+\beta}
\end{aligned}
$$


Without loss of generality, let $C_{2} \geq 1$. Then Eq. (21) becomes

$$
\begin{aligned}
\int_{0}^{t}\left\|D^{\beta} V^{*}\right\|_{E}^{2} d s \leq & \left(C_{0}\left(K_{1}+1\right)+C_{2} \exp (\nu t)\right) \varepsilon^{6} \\
& +C_{2} \exp (\nu t) \int_{0}^{t}\left(\sum_{i=1}^{4}\left\|D^{\beta} D_{x}^{i} V^{*}\right\|_{E}^{2}\right) d s \\
\leq & K_{2} \exp (\nu t) \varepsilon^{1+2 \beta}+C_{2} \exp (\nu t) \int_{0}^{t}\left(\sum_{i=1}^{4}\left\|D^{\beta+i} V^{*}\right\|_{E}^{2}\right) d s
\end{aligned}
$$

We claim that

$$
\begin{aligned}
\int_{0}^{t}\left\|D^{\beta} V^{*}\right\|_{E}^{2} d s \leq & K_{2} \exp (\nu t) \varepsilon^{4+2 \beta}\left(\sum_{k=0}^{n} C_{2}^{k} \exp (k \nu t) \varepsilon^{2 k} 2^{k}\right) \\
& +2^{n} C_{2}^{n+1} \exp ((n+1) \nu t) \int_{0}^{t}\left(\sum_{i=1}^{4}\left\|D^{\beta+n+i} V^{*}\right\|_{E}^{2}\right) d s
\end{aligned}
$$

We prove the claim by mathematical induction. When $n=0$, this is Eq. (34). Assume that Eq. (35) is true for $n=m$. By Eq. (34)

$$
\begin{aligned}
& \int_{0}^{t}\left\|D^{\beta+m+i} V^{*}\right\|_{E}^{2} d s \\
& \quad \leq\left(K_{2} \exp (\nu t) \varepsilon^{4+2 \beta+2 m+2 i}+C_{2} \exp (\nu t) \int_{0}^{t} \sum_{j=1}^{4}\left\|D^{\beta+m+i+j} V^{*}\right\|_{E}^{2} d s\right) .
\end{aligned}
$$

Then take $i=1$ to get

$$
\begin{aligned}
\int_{0}^{t} \sum_{i=1}^{4} & \left\|D^{\beta+m+i} V^{*}\right\|_{E}^{2} d s \\
\leq & K_{2} \exp (\nu t) \varepsilon^{4+2 \beta+2(m+1)} \\
& \quad+C_{2} \exp (\nu t) \int_{0}^{t} \sum_{j=1}^{4}\left\|D^{\beta+m+j+1} V^{*}\right\|_{E}^{2} d s+\sum_{i=2}^{4} \int_{0}^{t}\left\|D^{\beta+m+i} V^{*}\right\|_{E}^{2} d s \\
\leq & K_{2} \exp (\nu t) \varepsilon^{2 \beta+2(m+3)}+\left(C_{2} \exp (\nu t)+1\right) \sum_{i=1}^{4} \int_{0}^{t}\left\|D^{\beta+(m+1)+i} V^{*}\right\|_{E}^{2} d s .
\end{aligned}
$$


Thus

$$
\begin{aligned}
\int_{0}^{t}\left\|D^{\beta} V^{*}\right\|_{E}^{2} d s \leq & K_{2} \exp (\nu t) \varepsilon^{4+2 \beta}\left(\sum_{k=0}^{m} C_{2}^{k} \exp (k \nu t) \varepsilon^{2 k} 2^{k}\right) \\
& +2^{m} C_{2}^{m+1} \exp ((m+1) \nu t) \int_{0}^{t}\left(\sum_{i=1}^{4}\left\|D^{\beta+m+i} V^{*}\right\|_{E}^{2}\right) d s \\
\leq & K_{2} \exp (\nu t) \varepsilon^{4+2 \beta} \\
& \cdot\left(\sum_{k=0}^{m} C_{2}^{k} \exp (k \nu t) \varepsilon^{2 k} 2^{k}+2^{m} C_{2}^{m+1} \exp ((m+1) \nu t) \varepsilon^{2(m+1)}\right) \\
& +2^{m} C_{2}^{m+1} \exp ((m+1) \nu t) \cdot 2 C_{2} \exp (\nu t) \sum_{i=1}^{4} \int_{0}^{t}\left\|D^{\beta+(m+1)+i} V^{*}\right\|_{E}^{2} d s \\
\leq & K_{2} \exp (\nu t) \varepsilon^{4+2 \beta}\left(\sum_{k=0}^{m+1} C_{2}^{k} \exp (k \nu t) \varepsilon^{2 k} 2^{k}\right) \\
& +2^{m+1} C_{2}^{m+2} \exp ((m+2) \nu t) \sum_{i=1}^{4} \int_{0}^{t}\left\|D^{\beta+(m+1)+i} V^{*}\right\|_{E}^{2} d s .
\end{aligned}
$$

So Eq. (35) is true for $n=m+1$. Thus we have proved the claim.

From Eq. (8) we have

$$
\begin{aligned}
& \|V(t)\|^{2}+\cos \theta\|\eta(t)\|^{2}+\sigma\|\eta(t)\|_{H}^{2} \\
& \quad \leq \exp (M t)\left(\|V(0)\|^{2}+\cos \theta\|\eta(0)\|^{2}+\sigma\|\eta(0)\|_{H}^{2}\right)
\end{aligned}
$$

and

$$
\begin{aligned}
& \int_{0}^{t}\left(\|V(s)\|^{2}+\cos \theta\|\eta(s)\|^{2}+\sigma\|\eta(s)\|_{H}^{2}\right) d s \\
& \quad \leq M^{-1} \exp (M t)\left(\|V(0)\|^{2}+\cos \theta\|\eta(0)\|^{2}+\sigma\|\eta(0)\|_{H}^{2}\right) .
\end{aligned}
$$

From Eq. (7)

$$
\int_{0}^{t}\|V(s)\|_{E}^{2} d s \leq K_{3}\|V(0)\|^{2}+K_{4} \int_{0}^{t}\left(\|V(s)\|^{2}+\|\eta(s)\|^{2}+\left\|\eta_{x x}(s)\right\|^{2}\right) d s .
$$

Therefore, by Eqs. (36) and (37)

$$
\int_{0}^{t}\|V(s)\|_{E} d s \leq K_{5} \exp (M t)\left(\sum_{i=0}^{2}\left(\left\|D_{x}^{i} V(0)\right\|^{2}+\left\|D_{x}^{i} \eta(0)\right\|^{2}+\left\|D_{x}^{i} \eta(0)\right\|_{H}\right)\right) .
$$

By the same argument, for $\beta \geq 0$,

$$
\begin{aligned}
& \int_{0}^{t}\left\|D^{\beta} V(t)\right\|_{E} d s \\
& \quad \leq K_{5} \exp (M t)\left(\sum_{i=0}^{2}\left(\left\|D^{\beta} D_{x}^{i} V(0)\right\|^{2}+\left\|D^{\beta} D_{x}^{i} \eta(0)\right\|^{2}+\left\|D^{\beta} D_{x}^{i} \eta(0)\right\|_{H}\right)\right) .
\end{aligned}
$$


From the definition of $V^{*}$, we have

$$
\begin{aligned}
\left\|D^{\beta} V^{*}\right\|_{E} & \leq\left\|D^{\beta} V_{a}\right\|_{E}+\left\|D^{\beta} V_{i}\right\|_{E}+\left\|D^{\beta} V\right\|_{E} \\
& \leq\left\|D^{\beta} V\right\|_{E}+K_{6}\left(\sum_{i=0}^{5}\left\|D^{\beta} D_{x}^{i} \eta\right\|\right) .
\end{aligned}
$$

By Eqs. (37) and (39)

$$
\begin{aligned}
\int_{0}^{t}\left\|D^{\beta} V(t)\right\|_{E} d s & \leq K_{7} \exp (M t)\left(\sum_{i=0}^{6}\left(\left\|D^{\beta} D_{x}^{i} V(0)\right\|^{2}+\left\|D^{\beta} D_{x}^{i} \eta(0)\right\|^{2}\right)\right) \\
& \leq K_{8} \exp (M t) \varepsilon^{2(\beta-2)} .
\end{aligned}
$$

Let

$$
\varepsilon_{0}=\left(\left(2 C_{2}\right)^{-1} \exp (-\nu t)\right)^{1 / 2},
$$

where $C_{2}$ is the constant in Eq. (21). Then if $0<\varepsilon<\varepsilon_{0}$ and letting $r=\left(\varepsilon / \varepsilon_{0}\right)^{2}$, by Eqs. (35) and (39) we have

$$
\begin{aligned}
\int_{0}^{t}\left\|D^{\beta} V^{*}\right\|_{E}^{2} d s \leq & K_{2} \exp (\nu t) \varepsilon^{4+2 \beta}\left(1-r^{n+1}\right)(1-r)^{-1} \\
& +2^{n} C_{2}^{n+1} \exp ((n+1) \nu t) K_{8} \exp (M t)\left(\sum_{i=1}^{4} \varepsilon^{2(\beta+n+i-2)}\right) .
\end{aligned}
$$

Since $r^{n} \rightarrow 0$ as $n \rightarrow \infty$,

$$
\int_{0}^{t}\left\|D^{\beta} V^{*}\right\|_{E}^{2} d s \leq K_{2} \varepsilon^{4+2 \beta} \exp (\nu t)(1-r)^{-1} .
$$

This proves the lemma.

Lemma 5. If the condition in Lemma 4 is true, then

$$
\begin{aligned}
\left\|D^{i} \eta^{*}(t)\right\| & \leq \varepsilon^{2+i} L_{0} \exp (\delta t), \\
\left\|D^{i} \eta_{x x}(t)\right\| & \leq \varepsilon^{2+i} L_{0} \exp (\delta t) \quad \text { for } i=0,1,2, \\
\left\|V^{*}(t)\right\| & \leq \varepsilon^{2} L_{0} \exp (\delta t), \\
\left\|w^{*}(t)\right\| & \leq \varepsilon^{3} L_{0} \exp (\delta t),
\end{aligned}
$$

where $\eta^{*}=\eta-\eta_{1}$ and $L_{0}, \delta>0$ are constants depending only on $\theta, R, \sigma$.

Proof. By Eqs. (10) and (17), $\eta^{*}$ satisfies

$$
\begin{gathered}
\eta_{t}^{*}+A_{1} \eta_{x x}^{*}+B_{1} \eta_{x x x}^{*}+C_{1} \eta_{x x x x}^{*}=w^{*}, \\
\eta^{*}=0 \quad \text { at } t=0 .
\end{gathered}
$$

Multiply Eq. (41) by $\eta^{*}$, integrate over $[0, t] \times \Gamma$, and perform integration by parts 
to obtain

$$
\begin{gathered}
\left\|\eta^{*}(t)\right\|^{2}-2 A_{1} \int_{0}^{t}\left\|\eta_{x}^{*}\right\|^{2} d s+2 C_{1} \int_{0}^{t}\left\|\eta_{x x}^{*}\right\|^{2} d s \\
=2 \int_{0}^{t} d s \int_{\Gamma} w^{*} \eta^{*} d \Gamma \\
\leq 2 \int_{0}^{t} d s \int_{\Gamma}\left(\int_{0}^{1} w_{z}^{*} d z\right) \eta^{*} d \Gamma \\
\leq(a) \int_{0}^{t}\left\|\eta_{x}^{*}\right\|^{2} d s+\frac{1}{a} \int_{0}^{t}\left\|V^{*}\right\|^{2} d s,
\end{gathered}
$$

where $a$ is a constant, and choose $a=1$ so that

$$
\left\|\eta^{*}(t)\right\|^{2}+2 C_{1} \int_{0}^{t}\left\|\eta_{x x}^{*}\right\|^{2} d s \leq\left(2 A_{1}+1\right) \int_{0}^{t}\left\|\eta_{x}^{*}\right\|^{2} d s+\int_{0}^{t}\left\|V^{*}\right\|^{2} d s .
$$

By $\left\|\eta_{x}^{*}\right\|^{2} \leq\left(a_{1} / 2\right)\left\|\eta_{x x}^{*}\right\|^{2}+\left(1 / 2 a_{1}\right)\left\|\eta^{*}\right\|^{2}$, we have

$$
\left\|\eta^{*}(t)\right\|^{2} \leq\left(1+2 A_{1}\right)^{2}\left(8 C_{1}\right)^{-1} \int_{0}^{t}\left\|\eta^{*}(s)\right\|^{2} d s+\int_{0}^{t}\left\|V^{*}\right\|^{2} d s .
$$

Then by Lemma 4,

$$
\begin{aligned}
\left\|\eta^{*}(t)\right\|^{2} & \leq\left(8 C_{1}\right)\left(1+2 A_{1}\right)^{-2} \exp \left(\left(8 C_{1}\right)^{-1}\left(1+2 A_{1}\right)^{2} t\right) \int_{0}^{t}\left\|V^{*}(s)\right\|_{E}^{2} d s \\
& \leq L_{0} \varepsilon^{4} \exp \left(\left(\nu+\left(8 C_{1}\right)^{-1}\left(1+2 A_{1}\right)^{2}\right) t\right)(1-r)^{-1}
\end{aligned}
$$

for all $t \geq 0$. Multiply Eq. (17) by $\eta_{x x x x}$ and follow the same derivation as before to get

$$
\begin{aligned}
& \left\|\eta_{x x}(t)\right\|^{2}-\left\|\eta_{x x}(0)\right\|^{2}-2 A_{1} \int_{0}^{t}\left\|\eta_{x x x}(s)\right\|^{2} d s+2 C_{1} \int_{0}^{t}\left\|\eta_{x x x x}(s)\right\|^{2} d s \\
& =2 \int_{0}^{t} d s \int_{\Gamma} w^{*} \eta_{x x x x} d x
\end{aligned}
$$

and

$$
\left\|\eta_{x x}(t)\right\|^{2} \leq\left\|\eta_{x x}(0)\right\|^{2}+\int_{0}^{t}\left(A_{1}^{2} / C_{1}\right)\left\|\eta_{x x}\right\|^{2} d s+\left(1 / C_{1}\right) \int_{0}^{t}\left\|V^{*}\right\|_{E}^{2} d s,
$$

which implies

$$
\begin{aligned}
\left\|\eta_{x x}(t)\right\|^{2} \leq & \exp \left(\frac{A_{1}^{2} t}{C_{1}}\right)\left(\left\|\eta_{x x}(0)\right\|^{2}+\int_{0}^{t} \frac{1}{C_{1}}\left\|V^{*}\right\|_{E}^{2} d s\right) \\
& +\frac{1}{C_{1}} \int_{0}^{t}\left\|V^{*}\right\|_{E}^{2} d s \\
\leq & L_{0} \varepsilon^{4} \exp \left(\left(A_{1}^{2} C_{1}^{-1}+\nu\right) t\right)(1-r)^{-1}
\end{aligned}
$$

Furthermore when Eq. (33) was derived, we used $D_{x}^{i} V^{*}, i=1,2$, instead of $V^{*}$ to obtain

$$
\int_{0}^{t}\left\|D^{\beta} D_{x}^{t} V^{*}\right\|_{E}^{2} d s \leq C_{6} \varepsilon^{4+2(i+\beta)} \exp (\nu t)(1-r)^{-1} \quad \text { for all } t \geq 0 .
$$


Then similar to the derivations of Eqs. (43) and (44), we have

$$
\begin{aligned}
\left\|D_{x}^{i} \eta^{*}(t)\right\|^{2} & \leq L_{1} \varepsilon^{2(2+i)} \exp \left(\left(\nu+\left(8 C_{2}\right)^{-1}\left(1+2 A_{1}\right)^{2}\right) t\right)(1-r)^{-1}, \\
\left\|D_{x}^{i} \eta_{x x}(t)\right\|^{2} & \leq L_{1} \varepsilon^{2(2+i)} \exp \left(\left(A_{1}^{2} C_{1}^{-1}+\nu\right) t\right)(1-r)^{-1},
\end{aligned}
$$

for $i=1,2$ by A.3. From Eq. (22) and the derivation of Eq. (23), we have

$$
\begin{aligned}
& \left\|V^{*}(t)\right\|^{2}-\left\|V^{*}(0)\right\|^{2}+R^{-1} \int_{0}^{t}\left\|V^{*}\right\|_{E}^{2} d s \\
& \leq K_{5} \int_{0}^{t}\left\|V_{x}^{*}\right\|_{E}^{2} d s+K_{6} \int_{0}^{t}\left(\sum_{i=3}^{5}\left\|D_{x}^{i} \eta\right\|^{2}+\sum_{i=0}^{3}\left\|D_{t} D_{x}^{i} \eta\right\|^{2}\right)^{1 / 2} d s .
\end{aligned}
$$

By Eqs. (31) and (45), we have

$$
\left\|V^{*}(t)\right\|^{2}-\left\|V^{*}(0)\right\|^{2} \leq C_{11} \varepsilon^{6} \exp (\nu t)(1-r)^{-1}+C_{12} \varepsilon^{6} \exp (2 \nu t)(1-r)^{-1}
$$

and, by A.3,

$$
\begin{aligned}
\left\|V^{*}(t)\right\|^{2} & \leq\left\|V^{*}(0)\right\|^{2}+C_{11} \varepsilon^{6} \exp (\nu t)(1-r)^{-1}+C_{12} \varepsilon^{6} \exp (2 \nu t)(1-r)^{-1} \\
& \leq C_{13} \varepsilon^{4}+C_{11} \varepsilon^{6} \exp (\nu t)(1-r)^{-1}+C_{12} \varepsilon^{6} \exp (2 \nu t)(1-r)^{-1} \\
& \leq C_{14} \varepsilon^{4} \exp (2 \nu t)(1-r)^{-1}
\end{aligned}
$$

In exactly the same manner, we also have

$$
\left\|V_{x}^{*}(t)\right\|^{2} \leq C_{15} \varepsilon^{6}(2 \nu t)(1-r)^{-1}
$$

Since

$$
\left\|w^{*}(t)\right\|^{2}=\int_{-\infty}^{\infty} d x \int_{-1}^{0} d z\left(\int_{-1}^{z} w_{z}^{*} d z\right)^{2} \leq \int_{\Omega}\left(u_{z}^{*}\right)^{2} d A
$$

by (49),

$$
\left\|w^{*}(t)\right\|^{2} \leq C_{15} \varepsilon^{6} \exp (2 \nu t)(1-r)^{-1} .
$$

This completes the proof of Lemma 5 .

Now we are ready to prove Theorem 2.

Proof of Theorem 2. By Lemma 4, there exists an $\varepsilon_{0}=\left(\left(2 C_{2}\right)^{-1} \exp (-\nu T)\right)^{1 / 2}>0$ where $C_{2}$ and $\nu$ depend on $R, \theta$, and $\sigma$ only. If the initial data $V_{0}$ and $\eta_{0}$ satisfy (A.3) with $\varepsilon<\varepsilon_{0}$, then all the estimates in Lemma 5 hold. If we denote $\left(\int_{\Omega} u^{2} d A\right)^{1 / 2}$ by $\|u\|$, then by Lemma 5 for $0 \leq t \leq T$,

$$
\begin{aligned}
\left\|u-u_{b}\right\| & \leq\left\|\bar{u}_{1}(z) \eta^{*}\right\|+\left\|\bar{u}_{2}(z) \eta_{x}^{*}\right\|+\left\|u_{i}\right\|+\left\|u^{*}\right\| \\
& \leq L_{1} \varepsilon^{2} \exp (\delta t), \\
\left\|w-w_{b}\right\| & \leq\left\|\bar{w}_{1}(z) \eta_{x}^{*}\right\|+\left\|\bar{w}_{2}(z) \eta_{x x}^{*}\right\|+\left\|w_{i}\right\|+\left\|w^{*}\right\| \\
& \leq L_{1} \varepsilon^{3} \exp (\delta t),
\end{aligned}
$$

and

$$
\left\|\eta-\eta_{1}\right\|=\left\|\eta^{*}\right\| \leq L_{1} \varepsilon^{2} \exp (\delta t)
$$


where $L_{1}$ and $\delta$ only depend on $\theta, R, \sigma$. Therefore, $\left\{V_{b}, \eta_{1}\right\}$ is an asymptotic approximation for $0 \leq t \leq T$ in the $L_{2}$ norm to the exact solution $\{V, \eta\}$, and for $0 \leq t \leq T$

$$
u=u_{b}+O\left(\varepsilon^{2}\right), \quad w=\varepsilon w_{b}+O\left(\varepsilon^{3}\right), \quad \eta=\eta_{1}+O\left(\varepsilon^{2}\right),
$$

where $\varepsilon$ is a small positive parameter defined by the initial data. This proves the theorem.

Since all the estimates in Theorem 2 still hold if we replace $V, \eta, V_{b}$, and $\eta_{1}$ by $D^{\beta} V, D^{\beta} \eta, D^{\beta} V_{b}$, and $D^{\beta} \eta_{1}$ respectively, then by the usual Sobolev embedding theorem, we have the following corollary.

COROLlary. Theorem 2 also implies the following pointwise estimates for error bounds:

$$
\max _{-\infty<x<+\infty}\left|D^{\beta} \eta(t, x)-D^{\beta} \eta_{1}(t, x)\right| \leq M_{0} \varepsilon^{2} \exp (\delta t)
$$

for $0 \leq t \leq T$ and all $\beta$ in $0 \leq \beta \leq m$ where $m$ is a nonnegative integer and $\delta$, $M_{0}>0$ depending only on $R, \theta, \sigma$, and $m$.

Acknowledgment. The research reported here was supported in part by the National Science Foundation under grant CMS 8903083.

\section{REFERENCES}

[1] T. B. Benjamin, Wave formulation in laminar flow down an inclined plane, J. Fluid Mech. 2, 554-574 (1957)

[2] C. S. Yih, Stability of liquid flow down an inclined plane, Phys. Fluids 6, 321-334 (1963)

[3] C. C. Mei, Nonlinear gravity waves in a thin sheet of viscous fluid, J. Math. Phys. 45, 266-288 (1966)

[4] D. J. Benney, Long waves on liquid films, J. Math. Phys. 45, 150-155 (1966)

[5] D. D. Joseph, Fluid Dynamics of Viscoelastic Liquids, Springer-Verlag, New York, 1990

[6] S. M. Shih and M. C. Shen, Uniform asymptotic approximation for viscous fluid flow down an inclined plane, SIAM J. Math. Anal. 6, 560-582 (1975)

[7] A. Carasso and M. C. Shen, On viscous fluid flow down an inclined plane and the development of roll waves, SIAM J. Appl. Math. 33, 399-426 (1977)

[8] O. A. Ladyzhenskaya, The Mathematical Theory of Viscous Incompressible Flow, Gordon and Breach, New York, 1969

[9] S. M. Shih, Contributions to the theory of surface waves on a viscous fluid, Ph.D. Thesis, University of Wisconsin-Madison, 1973

[10] M. C. Shen, S. M. Sun, and R. E. Meyer, Surface waves on viscous magnetic fluid flow down an inclined plane, Phys. Fluids A 3, 439-445 (1991) 